\title{
ON THE NASH EQUILIBRIA FOR THE FCFS QUEUEING SYSTEM WITH LOAD-INCREASING SERVICE RATE
}

\author{
A. C. BROOMS, ${ }^{*}$ Birkbeck College
}

\begin{abstract}
We consider a service system $\left(Q_{\mathrm{S}}\right)$ that operates according to the first-come-first-served (FCFS) discipline, and in which the service rate is an increasing function of the queue length. Customers arrive sequentially at the system, and decide whether or not to join using decision rules based upon the queue length on arrival. Each customer is interested in selecting a rule that meets a certain optimality criterion with regard to their expected sojourn time in the system; as a consequence, the decision rules of other customers must be taken into account. Within a particular class of decision rules for an associated infiniteplayer game, the structure of the Nash equilibrium routeing policies is characterized. We prove that, within this class, there exist a finite number of Nash equilibria, and that at least one of these is nonrandomized. Finally, with the aid of simulation experiments, we explore the extent to which the Nash equilibria are characteristic of customer joining behaviour under a learning rule based on system-wide data.
\end{abstract}

Keywords: Queue; state-dependent service rate; noncooperative game; Nash equilibrium; simulation

2000 Mathematics Subject Classification: Primary 90B22; 91A10

Secondary 60E15; 91A13; 91A14

\section{Introduction}

This paper looks at customer joining behaviour in a first-come-first-served (FCFS) singleserver queueing system, where the service rate responds to changes in the queue size. Customers are prepared to join the system only if their expected time there is projected to be not too high. However, due to the nature of the service rate function, the routeing decisions of customers that arrive at the system in the future could affect the sojourn times of customers that are already present in the system.

In order to make an assessment as to whether or not quality-of-service requirements will be met upon joining such a system, assumptions regarding the form of the routeing decisions taken by other customers (as to whether to join or balk) will have to be made. The routeing decision to be made by each customer is whether or not to join the system on the basis of quantities that may depend on the number of customers observed on arrival at the system.

This leads us to analyse the problem as an infinite-player noncooperative (stationary) game, where the expected sojourn times at particular entry states are considered. Here, the aim is to characterize the conditions under which Nash equilibrium routeing policies exist, and to explore the structure of such policies.

Received 23 April 2004; revision received 7 November 2004.

* Postal address: School of Economics, Mathematics and Statistics, Birkbeck College, Malet Street, London WC1E 7HX, UK. Email address: a.brooms@bbk.ac.uk 
We also examine a scenario in which customers base their joining decisions on sample mean sojourn times, at particular entry states, of customers that have previously passed through the system. Thus, routeing decisions are subject to dynamic learning. Using simulation methods, we explore the extent to which the long-term (nontransient) behaviour of the system under the learning rule adheres to that under the Nash equilibria.

The seminal and most relevant work on the game-theoretic analysis of this class of queueing system was carried out by Altman and Shimkin [2], who investigated a processor-sharing system. They established the existence and uniqueness of a symmetric Nash equilibrium joining policy for the stationary game; it was also demonstrated, via simulation methods (and, in [1], using the theory of the stochastic approximations algorithm), that it can be used to characterize the convergent behaviour of the system (in an almost-sure sense) when customers base their joining decisions on a certain class of dynamic learning rule. (Later in the paper, we consider this learning rule for our system.) Buche and Kushner [6] analysed a modified learning rule for the processor-sharing system in which a discount factor was incorporated: this allowed the most recent system data to be weighted more heavily than was that from the distant past. Again, using theory related to stochastic approximations, they showed that their learning rule converges to that of the symmetric Nash equilibrium in a weak sense.

The general theory developed in [2] was applied to a multiple-server retrial system in [4], and to a FCFS system in which the service rate is nonincreasing in the system load in [5]. The analysis of the processor-sharing system was extended, in [3], to the case in which customers arrive at the system with differing quality-of-service requirements (although with the same exponential service distributions). Existence of a Nash equilibrium for this classheterogeneous scenario was established; uniqueness was also asserted, albeit under the proviso that the interarrival times were also independent and identically exponentially distributed (in order to facilitate a coupling argument).

The notion of individual optimality pertains neither to the processor-sharing system of [2] nor to the one considered in this paper, unless the routeing decisions of future arrivals are taken into account; this is because the sojourn time of a customer who enters such a system will depend on the joining rules adopted by future customers. Systems in which the characterization of an individually optimal policy is possible, without having to condition on the decision rules of others, were considered in [9] and [10], for example. Lippman and Stidham [8] also considered an exponential service system, consisting of a FCFS queue with a concave increasing, bounded service rate. However, they made the key assumption that a 'customer's holding time is not affected by future arrivals': thus, individual optimality can be characterized there.

The rest of the paper is organized as follows. In Section 2, we specify the model in detail, including a more thorough description of the decision rules used by the arriving customers. In Section 3, the generic random variables, and various processes defined with respect to these, will be introduced. In Section 4, we use coupling arguments to establish stochastic order results for the sojourn time in $Q_{\mathrm{S}}$ with respect to the entry state. This is followed up, in Section 5, by a discussion on monotonicity and continuity of the sojourn time with respect to symmetric threshold policies. The properties established in these latter two sections are then brought together in Section 6 to characterize the existence, and structure, of symmetric Nash equilibrium joining policies for the stationary game. An algorithm for finding the symmetric Nash equilibrium policies is outlined in Section 7. A simulation of the system under a similar learning rule to the one proposed in [2] is presented in Section 8. Plots, against time, of the empirical average sojourn times and of the entrance probabilities for various arrival states are presented, and we argue that these show a close correspondence to the behaviour under the stationary game when the Nash equilibrium is unique. We conclude our discussion in Section 9. 


\section{The model}

We let $\mathbb{Z}^{+}=\{1,2, \ldots\}, \mathbb{N}=\mathbb{Z}^{+} \cup\{0\}$, and $\mathbb{R}^{+}=\{x \in \mathbb{R}: x>0\}$ throughout the paper. An arriving customer has to choose between either joining a shared service system, which is a FCFS queue (denoted by $Q_{\mathrm{S}}$ ), or balking. It is assumed that $Q_{\mathrm{S}}$ has a buffer size $B$, which may be finite or infinite. Any customer who arrives when the buffer is full is not permitted to enter the system.

The departure process in $Q_{\mathrm{S}}$ at queue length $x$ forms a Poisson process at rate $\mu(x)$, where $\mu(x)$ is a strictly increasing, bounded function on $x \in\{1,2, \ldots, B\}$, with $\mu(0)=0$. We set $\bar{\mu}=\sup \{\mu(x), x=1,2, \ldots\}$.

Let $\theta$ be the quality-of-service requirement. If an arriving customer perceives that the (expected or empirical) sojourn time in $Q_{\mathrm{S}}$ is greater than this value, then it will be reluctant to enter the system. It is assumed that $\mu(1)^{-1}<\theta$. This condition ensures that it is always worthwhile for a customer to enter $Q_{\mathrm{S}}$ if the system is empty upon arrival.

Let the number of customers in $Q_{\mathrm{S}}$ at time $t$ be denoted by $X(t)$. Let $A_{k}$ be the arrival time of the $k$ th customer to the system, where $0=A_{0}<A_{1}<A_{2}<\cdots$; denote this $k$ th customer by the label $C_{k}, k \in \mathbb{N}$, where it is assumed that $C_{0}$ arrives at time $A_{0}$ (i.e. at time 0 ). We call the sequence of customers $C_{0}, C_{1}, \ldots, C_{k}, \ldots$ the (overall) arrival stream. The decision as to whether or not $C_{k}$ enters $Q_{\mathrm{S}}$ is taken on the basis of $X\left(A_{k}\right)$, the queue length in $Q_{\mathrm{S}}$ just prior to $C_{k}$ 's arrival.

A customer within the arrival stream can either be controlled or uncontrolled, with probability $1-p$ or $p$, respectively, independently of all other customers and irrespective of the state of the system upon arrival. If the customer that arrives at time $A_{k}$, say, is uncontrolled, then it will enter $Q_{\mathrm{S}}$ if and only if $X\left(A_{k}\right)<B$. We label the $r$ th controlled customer who could potentially arrive at (without necessarily entering) the system by $C^{(r)}, r \in \mathbb{Z}^{+}$.

We further define $T(r)$ to be the arrival index corresponding to the $r$ th controlled customer; within the overall arrival stream, the $r$ th controlled customer receives the label $C_{T(r)}$, and arrives at time $A_{T(r)}$. The precise construction of the function $T(\cdot): \mathbb{Z}^{+} \rightarrow \mathbb{N}$ will be given in Section 3.

A decision rule $u(\cdot):\{0,1, \ldots, B-1\} \rightarrow[0,1]$ is defined to be a function that specifies the probability that a customer obeying it enters $Q_{\mathrm{S}}$. This probability is equal to $u(x)$ if the number of customers in $Q_{\mathrm{S}}$ is equal to $x$ just prior to the customer's arrival. Let $\mathbb{U}$ denote the set of all such decision rules and define $u_{k}(\cdot)$ to be the decision rule associated with customer $C_{k}$, $k \in \mathbb{N}$, and $u^{(r)}(\cdot), r \in \mathbb{Z}^{+}$, to be the decision rule associated with the $r$ th controlled customer $C^{(r)}$. A policy $\pi=\left(u^{(1)}, u^{(2)}, \ldots, u^{(r)}, \ldots\right) \in \mathbb{U}^{\infty}$ is a collection of decision rules whose $r$ th member, $u^{(r)}(\cdot), r \in \mathbb{Z}^{+}$, represents the decision rule associated with $C^{(r)}$.

Let $v_{k}(x, \pi), x \in\{0,1, \ldots, B-1\}$, be the sojourn time of $C_{k}$ in $Q_{\mathrm{S}}$, given that $x$ customers were present in $Q_{\mathrm{S}}$ just prior to $C_{k}$ 's arrival and that any controlled customer arriving in the future adheres to its own decision rule, inferred from $\pi$. Furthermore, define $V_{k}(x, \pi)$ to be the expected value of $v_{k}(x, \pi)$. We draw attention to the slight abuse of terminology, insofar as $\pi$ need only represent the collection $\left(u^{\left(k^{\prime}+1\right)}, u^{\left(k^{\prime}+2\right)}, \ldots\right)$, where $k^{\prime}:=\max \{r \in \mathbb{N}: T(r) \leq k\}$ : for a given $x$, and by the assumption that $C_{k}$ joins $Q_{\mathrm{S}},\left(u^{(0)}, u^{(1)}, \ldots, u^{\left(k^{\prime}-1\right)}, u^{\left(k^{\prime}\right)}\right)$ does not provide any additional information about $v_{k}(x, \pi)$.

Also, let $v^{(k)}(x, \pi), x \in\{0,1, \ldots, B-1\}$, be the sojourn time of the $k$ th controlled customer to enter $Q_{\mathrm{S}}$, given that $x$ customers were present in $Q_{\mathrm{S}}$ just prior to the arrival of $C^{(k)}$ and that any controlled customer arriving in the future adheres to its own decision rule, inferred from $\pi$. Furthermore, we define $V^{(k)}(x, \pi)$ to be the expected value of $v^{(k)}(x, \pi)$. Here, $\pi$ need only represent $\left(u^{(k+1)}, u^{(k+2)}, \ldots\right)$. 
There is no collaboration between customers, and each controlled customer seeks to choose an optimal joining rule with regard to some measure of their projected sojourn time in $Q_{\mathrm{S}}$ and the quality-of-service requirement. Bearing these points in mind, we are led to analyse this system within the paradigm of the infinite-player noncooperative game.

A decision rule $u_{k}(\cdot)$ for the $k$ th customer in the overall arrival stream is said to be optimal against the policy $\pi$ if

$$
u_{k}(x)= \begin{cases}1 & \text { for } V_{k}(x, \pi)<\theta, \\ 0 & \text { for } V_{k}(x, \pi)>\theta, \\ q \in[0,1] & \text { for } V_{k}(x, \pi)=\theta,\end{cases}
$$

with $x \in\{0,1, \ldots, B-1\}$. The collection of all possible decision rules of $C_{k}$ that are optimal against $\pi$ is denoted by $\mathbb{U}_{k}(\pi)$, while a policy $\pi=\left(u^{(1)}, u^{(2)}, \ldots\right)$ is said to be a Nash equilibrium policy if, for every $r \in \mathbb{Z}^{+}$, the decision rule of the $r$ th controlled customer $u^{(r)}(\cdot)$ is optimal against $\pi$.

Under this regime, there is no guarantee that uncontrolled customers will ever exhibit optimal behaviour.

\section{Random variables and processes}

Let $\left\{M_{i}, i \in \mathbb{Z}^{+}\right\},\left\{N_{j}, j \in \mathbb{Z}^{+}\right\},\left\{U_{k}, k \in \mathbb{Z}^{+}\right\},\left\{U_{k}^{\phi}, k \in \mathbb{N}\right\}$, and $\left\{U_{l}^{\prime}, l \in \mathbb{Z}^{+}\right\}$be mutually independent sequences of random variables, where

- $\left\{M_{i}, i \in \mathbb{Z}^{+}\right\}$is a sequence of independent and identically distributed (i.i.d.) random variables with mean $0<\lambda^{-1}<\infty$;

- $\left\{A_{k}, k \in \mathbb{N}\right\}$, the sequence of arrival times to the system, is such that $A_{0}:=0$ and $A_{k}:=\sum_{i=1}^{k} M_{i}, k \in \mathbb{Z}^{+}$;

- $\left\{U_{k}, k \in \mathbb{N}\right\}$ is a sequence of i.i.d. random variables, uniformly distributed on the interval $(0,1]$, whose member $U_{k}$, for example, will be used to decide whether or not customer $C_{k}$ enters $Q_{\mathrm{S}}$;

- $\left\{U_{k}^{\phi}, k \in \mathbb{N}\right\}$ is a sequence of i.i.d. uniform random variables on $(0,1]$ used to determine whether an arrival to the system is controlled or uncontrolled;

- $\left\{N_{j}, j \in \mathbb{Z}^{+}\right\}$is a sequence of i.i.d. exponential random variables with mean $\bar{\mu}^{-1}<\infty$;

- $\left\{S_{l}, l \in \mathbb{Z}^{+}\right\}$, the sequence of potential departure times for customers in $Q_{\mathrm{S}}$, is such that $S_{l}:=\sum_{j=1}^{l} N_{j}$; and

- $\left\{U_{l}^{\prime}, l \in \mathbb{Z}^{+}\right\}$is a sequence of i.i.d. uniform random variables on $(0,1]$ used to determine whether a potential departure time corresponds to an actual departure or a dummy event.

We further define $\left\{t_{n}, n \in \mathbb{Z}^{+}\right\}$to be the order statistics for the set $\left\{A_{k}\right\} \cup\left\{S_{l}\right\}$, where $t_{i}<t_{j}$ for $i<j$.

The specifications of the arrival decisions and departures from $Q_{S}$ are presented at the end of the section. These will provide further motivation for the formal definitions of the stochastic processes, which are given next.

Definition 1. (Queue-length process.) For a given initial state $X(0)=x_{0}$ and policy $\pi$, let $\{X(t), t \geq 0\}$ be the queue-length process, where $X(t)$ represents the number of customers in 
the system at time $t$. This process is defined to be left continuous and piecewise constant, with its potential jumps described by the following relations, where $\mathbf{1}_{\{\cdot\}}$ is the indicator function:

$$
\begin{aligned}
X\left(A_{k}^{+}\right) & =X\left(A_{k}\right)+\mathbf{1}_{\left\{U_{k}<u_{k}\left(X\left(A_{k}\right)\right)\right\},}, & & k \in \mathbb{N}, \\
X\left(S_{l}^{+}\right) & =X\left(S_{l}\right)-\mathbf{1}_{\left\{U_{l}^{\prime}<\mu\left(X\left(S_{l}\right)\right) / \bar{\mu}\right\}}, & & l \in \mathbb{Z}^{+} .
\end{aligned}
$$

Note that if the $\left\{U_{l}\right\}$ were chosen to be uniform on the interval $[0,1]$, rather than on $(0,1]$, then we would also need to include $X\left(S_{l}\right)>0$ inside the indicator function in the second of the two relations.

Definition 2. (The remaining service transitions (RST) process.) Let $\{Z(t), t \geq 0\}$ be the RST process. This process is defined to be left continuous, piecewise constant, and nonincreasing, such that $Z(0)=X(0)=x_{0}$ and its potential jumps satisfy the following relation:

$$
Z\left(S_{l}^{+}\right)=Z\left(S_{l}\right)-\mathbf{1}_{\left\{Z\left(S_{l}\right)>0, U_{l}^{\prime}<\mu\left(X\left(S_{l}\right)\right) / \bar{\mu}\right\}}, \quad l \in \mathbb{Z}^{+} .
$$

When $C_{0}$ is in the queue, $Z(t)$ represents the number of customers present less those that are queueing behind $C_{0}$ (or the number of actual service transitions that still need to occur before $C_{0}$ exits) at time $t$, and $Z(t)=0$ if customer $C_{0}$ is not present at time $t$.

Definition 3. (Sojourn time.) If $C_{0}$ enters $Q_{\mathrm{S}}$ then we define its sojourn time to be

$$
v_{0}=\min \{t: Z(t)=0\} .
$$

\subsection{Arrivals at $Q_{S}$}

At time $A_{k}$, customer $C_{k}$ arrives at $Q_{\mathrm{S}}$ and enters the system with probability $\gamma$, say, which depends on its decision rule and the value of $X\left(A_{k}\right)$. The actual decision is based on the values of the random variable $U_{k}$ and $\gamma$ in the following way:

$$
C_{k} \text { enters } Q_{\mathrm{S}} \text { if and only if } U_{k} \leq \gamma .
$$

The mapping $T(\cdot): \mathbb{Z}^{+} \rightarrow \mathbb{N}$ is defined more precisely as follows:

$$
T(r)=\min \left\{n \in \mathbb{N}: \sum_{l=0}^{n} \mathbf{1}_{\left\{U_{l}^{\phi}>p\right\}}=r\right\}
$$

for $r \in \mathbb{Z}^{+}$. The set of indices corresponding to controlled customers in the overall arrival stream is

$$
\ell=\left\{m \in \mathbb{N}: \text { there exists an } r \in \mathbb{Z}^{+} \text {such that } m=T(r)\right\} .
$$

Thus, for $k \in \ell$ we have $u_{k}(x)=u^{\left(T^{-1}(k)\right)}(x)$, and for $k \notin \ell$ we have $u_{k}(x) \equiv 1$ for $x \in$ $\{0,1, \ldots, B-1\}$.

\subsection{Service at $Q_{\mathrm{S}}$}

For ease of exposition, define $x_{l}$ to be equal to $X\left(S_{l}\right)$, the queue length in $Q_{\mathrm{S}}$ just prior to a potential departure at time $S_{l}$. If $U_{l}^{\prime} \in\left(\mu\left(x_{l}\right) / \bar{\mu}, 1\right]$ then $S_{l}$ is considered to be a dummy service-completion instant; otherwise any customer at the server completes service and departs from the system.

The above procedure utilizes a uniformization technique (see [7]). The fact that such a procedure generates actual departure times, with the correct distribution, can be seen as follows. 
As long as the queue length remains at $x \in \mathbb{Z}^{+}$, the next potential departure is generated according to a Poisson process with rate $\mu(x)$. Now consider a Poisson process in which events occur at the uniform rate $\bar{\mu}$, the fastest rate at which departures could possibly occur. Whenever the queue length is $x$ and an event from the Poisson process with rate $\bar{\mu}$ occurs, it corresponds to an actual departure with probability $\mu(x) / \bar{\mu}$, independently of all other events. However, since this corresponds to a Bernoulli sampling of a Poisson process, departures at queue length $x$ are Poisson with rate $\bar{\mu} \times \mu(x) / \bar{\mu}=\mu(x)$, as anticipated.

\section{Monotonicity with respect to entry-queue size}

We show, in the sense of stochastic dominance, and in the sense of expectation, that $v_{0}(x, \pi)$ is an increasing function of $x$ for any $\pi$ that is a member of a certain class of policies. This class is defined as follows.

Definition 4. Let $\mathbb{T}$ be the class of decision rules that are nonincreasing functions of the queue length $x \in\{0,1, \ldots, B-1\}$. Also, let $\mathbb{T}^{\infty}$ be the class of policies in which the decision rule for each controlled customer is a member of $\mathbb{T}$. We note that the decision rule corresponding to an uncontrolled customer trivially belongs to $\mathbb{T}$; thus, with regard to the proofs in this section, no special distinction need be made between controlled and uncontrolled customers.

Evaluating the distribution of $v_{0}(x, \pi)$ appears to be less than straightforward in any but the simplest cases. This difficulty will be circumvented by using stochastic coupling and forward induction techniques. The collections of random variables and stochastic processes (to which we shall loosely refer as 'systems') upon which these stochastic comparisons will be based are introduced next.

Definition 5. (System $\mathcal{X}$.) This system is characterized by the sets of random variables, decision rules, and stochastic processes listed below:

(I) $\mathcal{M}=\left\{M_{i}\right\}, \mathcal{N}=\left\{N_{j}\right\}, \mathcal{U}=\left\{U_{k}\right\}, \mathcal{U}^{\phi}=\left\{U_{k}^{\phi}\right\}$, and $\mathcal{U}^{\prime}=\left\{U_{l}^{\prime}\right\} ;$

(II) the arrival time sequence $\mathcal{A}=\left\{A_{k}\right\}$ and the potential departure time sequence $\delta=\left\{S_{l}\right\}$;

(III) the rule that customer $C_{0}$ enters $Q_{\mathrm{S}}$ at time $A_{0}=0$, with all other controlled customers adhering to policy $\pi \in \mathbb{T}^{\infty}$;

(IV) the queue-length process $\{X(t), t \geq 0\}$, with $X(0)=x$;

(V) the RST process $\{Z(t), t \geq 0\}$, with $Z(0)=x$; and

(VI) $v_{0}$, the sojourn time of $C_{0}$ in $Q_{\mathrm{S}}$.

Definition 6. (System $\tilde{X}$.) This system is characterized in a similar way to $\mathcal{X}$, except that the

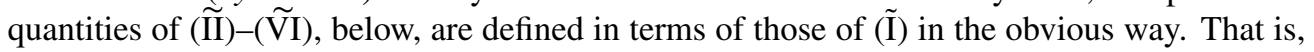
the system depends on

(I) $\tilde{\mathcal{M}}=\left\{\tilde{M}_{i}\right\}, \tilde{\mathcal{N}}=\left\{\tilde{N}_{j}\right\}, \tilde{U}=\left\{\tilde{U}_{k}\right\}, \tilde{U}^{\phi}=\left\{\tilde{U}_{k}^{\phi}\right\}$, and $\tilde{U}^{\prime}=\left\{\tilde{U}_{l}^{\prime}\right\}$, which have the same distributions as $\mathcal{M}, \mathcal{N}, U, \mathcal{U}^{\phi}$, and $\mathcal{U}^{\prime}$, respectively;

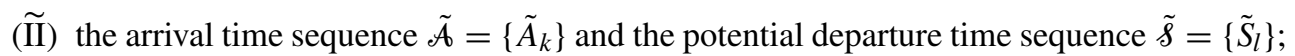

(III) the same rule as in (III) for $\mathcal{X}$, with $\tilde{A}_{0}=0$;

(IV) the queue-length process $\{\tilde{X}(t), t \geq 0\}$, with $\tilde{X}(0)=x+1$; 
$(\tilde{\mathrm{V}})$ the RST process $\{\tilde{Z}(t), t \geq 0\}$, with $\tilde{Z}(0)=x+1$; and

( $\widetilde{\mathrm{VI}}) \tilde{v}_{0}$, the sojourn time of $C_{0}$ in $Q_{\mathrm{S}}$.

We intend to relate $\mathcal{X}$ to $\tilde{X}$ using the following device.

Definition 7. (Coupling $\mathcal{C}$.) Set

$$
\begin{aligned}
M_{i} & =\tilde{M}_{i}, & & i \in \mathbb{Z}^{+}, \\
N_{j} & =\tilde{N}_{j}, & & j \in \mathbb{Z}^{+}, \\
U_{k} & =\tilde{U}_{k}, & & k \in \mathbb{N}, \\
U_{k}^{\phi} & =\tilde{U}_{k}^{\phi}, & & k \in \mathbb{N}, \\
U_{l}^{\prime} & =\tilde{U}_{l}^{\prime}, & & l \in \mathbb{Z}^{+} .
\end{aligned}
$$

The effect of this procedure is that, under $\mathcal{X}$, the arrival instants $\left\{A_{k}\right\}$, the potential departure times $\left\{S_{l}\right\}$, the $\left\{U_{k}\right\}$, the positions of controlled customers in the overall arrival sequence, and the $\left\{U_{l}^{\prime}\right\}$ take the same values as their counterparts under $\tilde{X}$, at each realization.

The next result allows us to infer that, under the above coupling, $v_{0} \leq \tilde{v}_{0}$.

Lemma 1. For the systems $\mathcal{X}$ and $\tilde{X}$ under coupling $\mathcal{C}$, in which $\pi \in \mathbb{T}^{\infty}$, one of the following sets of relations will hold at each time $t \in \mathbb{R}^{+}$:

$$
\begin{aligned}
X(t)+1 & =\tilde{X}(t), \\
Z(t)+1 & =\tilde{Z}(t), \\
X(t) & =\tilde{X}(t), \\
Z(t) & =\tilde{Z}(t), \\
X(t) & =\tilde{X}(t), \\
Z(t)+1 & =\tilde{Z}(t) .
\end{aligned}
$$

Proof. By definition of $\mathcal{X}$ and $\tilde{X}$,

$$
Z\left(0^{+}\right)=X\left(0^{+}\right)=x+1<x+2=\tilde{X}\left(0^{+}\right)=\tilde{Z}\left(0^{+}\right)
$$

Assume that $t_{n+1}$ corresponds to an arrival with $t_{n+1}=A_{r}$, or a departure with $t_{n+1}=S_{m}$, such that $C_{0}$ is still present in $Q_{\mathrm{S}}$ under $\mathcal{X}$. Before proceeding, we observe that if $t_{n+1}$ does indeed correspond to an arrival time then, due to the class of policies and the coupling being considered, one of the following three scenarios must arise:

(i) $C_{r}$ enters $Q_{\mathrm{S}}$ under both $\mathcal{X}$ and $\tilde{X}$,

(ii) $C_{r}$ enters $Q_{\mathrm{S}}$ under $\mathcal{X}$ only, or

(iii) $C_{r}$ balks under both $\mathcal{X}$ and $\tilde{X}$.

Case 1: Suppose that (1) holds at time $t_{n}^{+}$. First, we assume that $t_{n+1} \in\left\{A_{k}\right\}$. Then, under (i),

$$
X\left(t_{n+1}^{+}\right)=X\left(t_{n}^{+}\right)+1=\tilde{X}\left(t_{n}^{+}\right)=\tilde{X}\left(t_{n+1}^{+}\right)-1
$$


under (ii),

$$
X\left(t_{n+1}^{+}\right)=X\left(t_{n}^{+}\right)+1=\tilde{X}\left(t_{n}^{+}\right)=\tilde{X}\left(t_{n+1}^{+}\right)
$$

and, under (iii), the states of the queue-length processes for $\mathcal{X}$ and $\tilde{X}$ at time $t_{n+1}^{+}$are the same as they were at time $t_{n}^{+}$. Also, since $C_{r}$ would queue behind $C_{0}$ if it were to enter $Q_{\mathrm{S}}$, there would be no change in the RST processes in each of the above scenarios, i.e.

$$
Z\left(t_{n+1}^{+}\right)=Z\left(t_{n}^{+}\right)=\tilde{Z}\left(t_{n}^{+}\right)-1=\tilde{Z}\left(t_{n+1}^{+}\right)-1 .
$$

Thus, at time $t_{n+1}^{+}$, (1) holds under scenarios (i) and (iii), whereas (3) holds under scenario (ii).

Now assume that $t_{n+1} \in\left\{S_{l}\right\}$. Since $x_{m}<\tilde{x}_{m}$ by assumption, we have $\mu\left(x_{m}\right)<\mu\left(\tilde{x}_{m}\right)$. If $U_{m}^{\prime} \leq \mu\left(x_{m}\right) / \bar{\mu}$ then an actual departure occurs under both $\mathcal{X}$ and $\tilde{X}$. Therefore,

$$
\begin{aligned}
& X\left(t_{n+1}^{+}\right)=X\left(t_{n}^{+}\right)-1=\left(\tilde{X}\left(t_{n}^{+}\right)-1\right)-1=\tilde{X}\left(t_{n+1}^{+}\right)-1, \\
& Z\left(t_{n+1}^{+}\right)=Z\left(t_{n}^{+}\right)-1=\left(\tilde{Z}\left(t_{n}^{+}\right)-1\right)-1=\tilde{Z}\left(t_{n+1}^{+}\right)-1 .
\end{aligned}
$$

Thus, (1) holds at time $t_{n+1}^{+}$. If $U_{m}^{\prime} \in\left(\mu\left(x_{m}\right) / \bar{\mu}, \mu\left(\tilde{x}_{m}\right) / \bar{\mu}\right]$ then an actual departure occurs under $\tilde{X}$ but not under $\mathcal{X}$. Therefore,

$$
\begin{aligned}
& X\left(t_{n+1}^{+}\right)=X\left(t_{n}^{+}\right)=\tilde{X}\left(t_{n}^{+}\right)-1=\tilde{X}\left(t_{n+1}^{+}\right), \\
& Z\left(t_{n+1}^{+}\right)=Z\left(t_{n}^{+}\right)=\tilde{Z}\left(t_{n}^{+}\right)-1=\tilde{Z}\left(t_{n+1}^{+}\right) .
\end{aligned}
$$

Thus, (2) holds at time $t_{n+1}^{+}$. Finally, if $U_{m}^{\prime}>\mu\left(\tilde{x}_{m}\right) / \bar{\mu}$ then the states of the processes remain unchanged, i.e.

$$
\begin{aligned}
& X\left(t_{n+1}^{+}\right)=X\left(t_{n}^{+}\right)=\tilde{X}\left(t_{n}^{+}\right)-1=\tilde{X}\left(t_{n+1}^{+}\right)-1, \\
& Z\left(t_{n+1}^{+}\right)=Z\left(t_{n}^{+}\right)=\tilde{Z}\left(t_{n}^{+}\right)-1=\tilde{Z}\left(t_{n+1}^{+}\right)-1,
\end{aligned}
$$

meaning that (1) holds at time $t_{n+1}^{+}$.

Case 2: Suppose that (2) holds at time $t_{n}^{+}$. It follows that (2) holds at time $t_{n+1}^{+}$also, as the following argument shows. Again, we first assume that $t_{n+1} \in\left\{A_{k}\right\}$.

Since the queue lengths are identical under both $\mathcal{X}$ and $\tilde{X}$, as are the decision rules for $C_{r}$, the decision as to whether or not to enter $Q_{\mathrm{S}}$ will be the same under both systems. Hence,

$$
X\left(t_{n+1}^{+}\right)=\tilde{X}\left(t_{n+1}^{+}\right) \text {. }
$$

Again, since $C_{r}$ would queue behind $C_{0}$ were it actually to enter $Q_{\mathrm{S}}$, the states of the RST processes remain unchanged.

Now assume that $t_{n+1} \in\left\{S_{l}\right\}$. Since $x_{m}=\tilde{x}_{m}$, we have $\mu\left(x_{m}\right)=\mu\left(\tilde{x}_{m}\right)$. If $U_{m}^{\prime} \leq \mu\left(x_{m}\right) / \bar{\mu}$ then there is an actual departure under both $\mathcal{X}$ and $\tilde{X}$, and so

$$
\begin{aligned}
& X\left(t_{n+1}^{+}\right)=X\left(t_{n}^{+}\right)-1=\tilde{X}\left(t_{n}^{+}\right)-1=\tilde{X}\left(t_{n+1}^{+}\right), \\
& Z\left(t_{n+1}^{+}\right)=Z\left(t_{n}^{+}\right)-1=\tilde{Z}\left(t_{n}^{+}\right)-1=\tilde{Z}\left(t_{n+1}^{+}\right) .
\end{aligned}
$$

If $U_{m}^{\prime}>\mu\left(x_{m}\right) / \bar{\mu}$ then there are no actual departures under either $\mathcal{X}$ or $\tilde{X}$, and so there is no change in either the queue-length processes or the RST processes, i.e.

$$
\begin{aligned}
& X\left(t_{n+1}^{+}\right)=X\left(t_{n}^{+}\right)=\tilde{X}\left(t_{n}^{+}\right)=\tilde{X}\left(t_{n+1}^{+}\right), \\
& Z\left(t_{n+1}^{+}\right)=Z\left(t_{n}^{+}\right)=\tilde{Z}\left(t_{n}^{+}\right)=\tilde{Z}\left(t_{n+1}^{+}\right) .
\end{aligned}
$$


Case 3: Suppose that (3) holds at time $t_{n}^{+}$. It follows that (3) holds at time $t_{n+1}^{+}$also, as the argument below shows. Once more, first assume that $t_{n+1} \in\left\{A_{k}\right\}$.

For the same reasons as in the previous case, the queue lengths remain equal, i.e.

$$
X\left(t_{n+1}^{+}\right)=\tilde{X}\left(t_{n+1}^{+}\right)
$$

and there are no changes in the RST processes.

Finally, assume that $t_{n+1} \in\left\{S_{l}\right\}$. Since $x_{m}=\tilde{x}_{m}$, we have $\mu\left(x_{m}\right)=\mu\left(\tilde{x}_{m}\right)$. Again, for the same reasons as in the previous case, if $U_{m}^{\prime} \leq \mu\left(x_{m}\right) / \bar{\mu}$ then

$$
\begin{aligned}
& X\left(t_{n+1}^{+}\right)=X\left(t_{n}^{+}\right)-1=\tilde{X}\left(t_{n}^{+}\right)-1=\tilde{X}\left(t_{n+1}^{+}\right) \\
& Z\left(t_{n+1}^{+}\right)=Z\left(t_{n}^{+}\right)-1=\left(\tilde{Z}\left(t_{n}^{+}\right)-1\right)-1=\tilde{Z}\left(t_{n+1}^{+}\right)-1 .
\end{aligned}
$$

On the other hand, if $U_{m}^{\prime}>\mu\left(x_{m}\right) / \bar{\mu}$ then there is no change in either the queue-length processes or the RST processes.

Recalling the definition of the sojourn time of $C_{0}$ in $Q_{\mathrm{S}}$, we now have the following lemma.

Lemma 2. For all $\pi \in \mathbb{T}^{\infty}$ and $k \in \mathbb{N}, V_{k}(x, \pi)$ is strictly increasing in $x$, in the sense that, for $x \in\{0,1, \ldots, B-2\}$, there exist constants $\left\{\delta_{x}\right\}$ such that

$$
V_{k}(x+1, \pi)-V_{k}(x, \pi) \geq \delta_{x}>0
$$

uniformly in $\pi$.

Proof. Without loss of generality, and to be specific, consider customer $C_{0}$ and the systems $\mathcal{X}$ and $\tilde{X}$ under coupling $\mathcal{C}$. From the definitions of $v_{0}$ and $\tilde{v}_{0}$, and by Lemma $1, v_{0} \leq \tilde{v}_{0}$, which implies that $\mathrm{E}\left[v_{0}\right] \leq \mathrm{E}\left[\tilde{v}_{0}\right]$. To establish the sharp inequality, define the event $D_{x}$ :

$$
D_{x}=\left\{S_{x+1}<A_{1}, U_{m}^{\prime} \leq \mu\left(x_{m}\right) / \bar{\mu}, m=1, \ldots, x+1\right\} .
$$

This is the event that customer $C_{1}$ arrives no earlier than the $(x+1)$ th departure under both systems, where $C_{0}$ leaves under $\mathcal{X}$ at time $S_{x+1}$ but becomes the only customer left in $Q_{\mathrm{S}}$ under $\tilde{X}$ at that time. By conditioning on this event, and noting that $v_{0}<\tilde{v}_{0}$ on $D_{x}$ and that $\mathrm{P}\left(D_{x}\right)>0$, the result follows.

\section{Monotonicity and continuity with respect to threshold policies}

In this section, we examine the behaviour of the sojourn time in $Q_{\mathrm{S}}$ with respect to a certain type of threshold rule, which is introduced below.

Definition 8. For $L \in \mathbb{N}$ and $q \in[0,1]$, an $[L, q]$-threshold decision rule $u(\cdot)$ is defined as follows:

$$
u(x)= \begin{cases}1 & \text { if } x<L, \\ q & \text { if } x=L, \\ 0 & \text { if } x>L .\end{cases}
$$

This may be represented more compactly by $[L, q]$, or indeed $[g]$, where $g=L+q$. Of course, for $B<\infty,[B]$ is equivalent to $[g]$ whenever $g>B$. 
We are ultimately interested in the characterization of symmetric policies: these are policies in which every controlled customer adopts the same decision rule. A policy $\pi$ in which the decision rule for each controlled customer is given by $[g]$ is denoted by $[g]^{\infty}$. We call this a symmetric threshold policy.

We next introduce a further two systems, which will facilitate the proofs of the results of this section.

Definition 9. (System g.) For this system, (I), (II), (IV), (V), and (VI) are exactly as in the definition of $\mathcal{X}$. However, (III) becomes

(III) the rule that customer $C_{0}$ enters $Q_{\mathrm{S}}$ at time $A_{0}=0$, with all other controlled customers adhering to policy $[g]^{\infty}, g \in[0, B)$.

Definition 10. (System $\tilde{g}_{.}$) For this system, $(\tilde{\mathrm{I}}),(\widetilde{\mathrm{II}})$, and $(\widetilde{\mathrm{VI}})$ are precisely the same as in the definition of $\tilde{\mathcal{X}}$. However, the system is further characterized by

(III) the rule that customer $C_{0}$ enters $Q_{\mathrm{S}}$ at time $\tilde{A}_{0}=0$, with all other controlled customers adhering to the policy $[\tilde{g}]^{\infty}, g<\tilde{g} \leq B$ (where the latter inequality is strict if $B=\infty$ );

(IV) the queue-length process $\{\tilde{X}(t), t \geq 0\}$, with $\tilde{X}(0)=x$; and

$(\tilde{\mathrm{V}})$ the $\operatorname{RST}$ process $\{\tilde{Z}(t), t \geq 0\}$, with $\tilde{Z}(0)=x$.

It is implicit from the definitions of $g$ and $\tilde{g}$ that $L<B$. Note that, from now on, $g \in[0, B]$ will be taken to mean that $0 \leq g \leq B$ when $B$ is finite, and that $0 \leq g<B$ when $B$ is infinite, unless specified to the contrary.

The next two results will be used to infer results about $V_{k}\left(\cdot,[g]^{\infty}\right)$ on the intervals $[0,1]$ and $[1, B]$.

Lemma 3. For the systems $g$ and $\tilde{g}$ under coupling $\mathcal{C}$, the following set of relations holds at each time $t \in \mathbb{R}^{+}$:

$$
\begin{aligned}
X(t) & \leq \tilde{X}(t), \\
Z(t) & \geq \tilde{Z}(t) .
\end{aligned}
$$

Proof. Assume that $t_{n+1}$ corresponds to an arrival time with $t_{n+1}=A_{r}$, or to a potential departure time with $t_{n+1}=S_{m}$, such that $C_{0}$ is still present in $Q_{\mathrm{S}}$ under $\tilde{g}_{\text {. }}$.

First note that

$$
Z\left(0^{+}\right)=X\left(0^{+}\right)=x+1=\tilde{X}\left(0^{+}\right)=\tilde{Z}\left(0^{+}\right) .
$$

Now suppose that

$$
\begin{aligned}
& X\left(t_{n}^{+}\right) \leq \tilde{X}\left(t_{n}^{+}\right), \\
& Z\left(t_{n}^{+}\right) \geq \tilde{Z}\left(t_{n}^{+}\right) .
\end{aligned}
$$

Case 1: Relation (6) is strict. First assume that $t_{n+1} \in\left\{A_{k}\right\}$. If $\tilde{X}\left(t_{n}^{+}\right)<B$ then $C_{r}$ enters $Q_{\mathrm{S}}$ under neither, one, or both of $g$ and $\tilde{g}$ (noting that the last of these scenarios certainly occurs if $C_{r}$ is uncontrolled); it follows that

$$
X\left(t_{n+1}^{+}\right) \leq \tilde{X}\left(t_{n+1}^{+}\right)
$$

(which holds with equality when $C_{r}$ enters $Q_{\mathrm{S}}$ under $g$ only and $X\left(t_{n}^{+}\right)=\tilde{X}\left(t_{n}^{+}\right)-1$ ). If $\tilde{X}\left(t_{n}^{+}\right)=B$ then $C_{r}$ can only enter $Q_{\text {S }}$ under $g$ (and will certainly enter under $g$ if $C_{r}$ is uncontrolled). Hence,

$$
X\left(t_{n+1}^{+}\right) \leq \tilde{X}\left(t_{n+1}^{+}\right)=B .
$$


Since $C_{r}$ can never queue ahead of $C_{0}$ in any of these scenarios, there can be no change in the RST processes, meaning that

$$
Z\left(t_{n+1}^{+}\right)=Z\left(t_{n}^{+}\right) \geq \tilde{Z}\left(t_{n}^{+}\right)=\tilde{Z}\left(t_{n+1}^{+}\right) .
$$

Now assume that $t_{n+1} \in\left\{S_{l}\right\}$. Since $x_{m}<\tilde{x}_{m}$, we have $\mu\left(x_{m}\right)<\mu\left(\tilde{x}_{m}\right)$. If $U_{m}^{\prime} \leq \mu\left(x_{m}\right) / \bar{\mu}$ then an actual departure occurs under both $g$ and $\tilde{g}$, and so

$$
\begin{aligned}
& X\left(t_{n+1}^{+}\right)=X\left(t_{n}^{+}\right)-1<\tilde{X}\left(t_{n}^{+}\right)-1=\tilde{X}\left(t_{n+1}^{+}\right), \\
& Z\left(t_{n+1}^{+}\right)=Z\left(t_{n}^{+}\right)-1 \geq \tilde{Z}\left(t_{n}^{+}\right)-1=\tilde{Z}\left(t_{n+1}^{+}\right) .
\end{aligned}
$$

If $U_{m}^{\prime} \in\left(\mu\left(x_{m}\right) / \bar{\mu}, \mu\left(\tilde{x}_{m}\right) / \bar{\mu}\right]$ then an actual departure occurs under $\tilde{g}$ but not under $g$, and so

$$
\begin{aligned}
& X\left(t_{n+1}^{+}\right)=X\left(t_{n}^{+}\right) \leq \tilde{X}\left(t_{n}^{+}\right)-1=\tilde{X}\left(t_{n+1}^{+}\right), \\
& Z\left(t_{n+1}^{+}\right)=Z\left(t_{n}^{+}\right) \geq \tilde{Z}\left(t_{n}^{+}\right)>\tilde{Z}\left(t_{n}^{+}\right)-1=\tilde{Z}\left(t_{n+1}^{+}\right) .
\end{aligned}
$$

If $U_{m}^{\prime}>\mu\left(x_{m}\right) / \bar{\mu}$ then there is no change, i.e. (4) holds strictly, and (5) also holds at time $t_{n+1}^{+}$.

Case 2: Relation (6) holds with equality. First assume that $t_{n+1} \in\left\{A_{k}\right\}$. Now $C_{r}$ enters $Q_{\text {S }}$ under neither or both of $g_{\text {and }} \tilde{g}$, or just $\tilde{g}$ alone (and the second of these scenarios will certainly hold if $X\left(t_{n}^{+}\right)=\tilde{X}\left(t_{n}^{+}\right)<B$ and $C_{r}$ is uncontrolled). Therefore,

$$
X\left(t_{n+1}^{+}\right) \leq \tilde{X}\left(t_{n+1}^{+}\right) \text {. }
$$

As in the previous case, $C_{r}$ is unable to queue ahead of $C_{0}$ and, so, (5) again holds at time $t_{n+1}^{+}$.

Finally, assume that $t_{n+1} \in\left\{S_{l}\right\}$. Since $x_{m}=\tilde{x}_{m}$, we have $\mu\left(x_{m}\right)=\mu\left(\tilde{x}_{m}\right)$. If $U_{m}^{\prime} \leq$ $\mu\left(x_{m}\right) / \bar{\mu}$ then an actual departure occurs under both $g$ and $\tilde{g}$, and so

$$
\begin{aligned}
& X\left(t_{n+1}^{+}\right)=X\left(t_{n}^{+}\right)-1=\tilde{X}\left(t_{n}^{+}\right)-1=\tilde{X}\left(t_{n+1}^{+}\right), \\
& Z\left(t_{n+1}^{+}\right)=Z\left(t_{n}^{+}\right)-1 \geq \tilde{Z}\left(t_{n}^{+}\right)-1=\tilde{Z}\left(t_{n+1}^{+}\right) .
\end{aligned}
$$

If $U_{m}^{\prime}>\mu\left(x_{m}\right) / \bar{\mu}$ then there is no change, i.e. (4) holds with equality, and (5) holds at time $t_{n+1}^{+}$. Lemma 4. For the systems $g$ and $\tilde{g}$ under coupling $\mathcal{C}$, with $0<g<\tilde{g} \leq 1$, the following set of relations holds at each time $t \in \mathbb{R}^{+}$throughout the sojourn of $C_{0}$ (in either system):

$$
\begin{aligned}
& X(t)=\tilde{X}(t), \\
& Z(t)=\tilde{Z}(t) .
\end{aligned}
$$

Proof. Since $X(0)=x=\tilde{X}(0)$, arrivals into $Q_{S}$, actual departures, and dummy events will coincide in the two systems during the sojourn of $C_{0}$, at least until such time that a disparity in the arrival decisions occurs. However, since $0<g<\tilde{g} \leq 1$, the first opportunity for a customer to enter $Q_{\mathrm{S}}$ under $\tilde{g}$, but not under $g$, is when the queues are completely empty, and $C_{0}$ will obviously have left by this time. Therefore, as a result, the relations (7) and (8) hold.

Next, we define the following quantity for the ensuing discussion, where $[\tilde{g}]=[\tilde{L}, \tilde{q}]$ :

$$
\hat{q}:=1-(1-\tilde{q}) \mathbf{1}_{\{L=\tilde{L}\}} .
$$


Lemma 5. For each $k \in \mathbb{N}$ and $x \in\{0,1, \ldots, B-1\}$,

(i) $V_{k}\left(x,[g]^{\infty}\right)$ is constant in $g$ on $[0,1]$ and

(ii) $V_{k}\left(x,[g]^{\infty}\right)$ is strictly decreasing in $g$ on $[1, B]$.

Proof. Without loss of generality, and to be specific, consider customer $C_{0}$ and the systems $g$ and $\tilde{g}$ under coupling $\mathcal{C}$. From the definitions of $v_{0}$ and $\tilde{v}_{0}$, and by invoking Lemma 3 , it is easy to see that

$$
\mathrm{E}\left[v_{0}\right] \geq \mathrm{E}\left[\tilde{v}_{0}\right]
$$

which holds with equality whenever $0 \leq g<\tilde{g} \leq 1$, by Lemma 4 , thereby establishing part (i). We thus assume that $1 \leq g<\tilde{g}$ and define the following events: for $x<L$,

$$
\begin{aligned}
F_{\alpha}=\left\{A_{L-x}<S_{1} ; A_{L-x+1}>S_{x+1} ; U_{L-x}^{\phi}>p ; U_{L-x} \in(q, \hat{q}] ;\right. \\
\\
U_{1}^{\prime} \in\left(\mu\left(X\left(S_{1}\right)\right) / \bar{\mu}, \mu\left(\tilde{X}\left(S_{1}\right)\right) / \bar{\mu}\right] ; \\
\left.U_{m}^{\prime} \leq \mu\left(X\left(S_{m}\right)\right) / \bar{\mu}, m=2, \ldots, x+1\right\} .
\end{aligned}
$$

Any realization of the systems on $F_{\alpha}$ under coupling $\mathcal{C}$ results in the following occurrences, in the order presented.

1. $L-x-1$ customers enter $Q_{\mathrm{S}}$ under both $g$ and $\tilde{g}$, resulting in the queue size increasing to $L$ and $C_{0}$ remaining at position $x+1$ in both cases.

2. A controlled customer enters $Q_{\mathrm{S}}$ under $\tilde{g}$ but not under $g$. This results in the queue size under $\tilde{g}$ increasing from $L$ to $L+1$, but remaining at $L$ under $g$, with $C_{0}$ still at position $x+1$ in both cases.

3. A departure occurs under $\tilde{g}$ but not under $g$. This results in the queue size being equal to $L$ in both cases, $C_{0}$ remaining at position $x+1$ under $g$, and $C_{0}$ moving to position $x$ (or indeed exiting the system if $x=0$ ) under $\tilde{g}$.

4. A further $x$ departures occur under both systems (before the next arrival), resulting in $C_{0}$ leaving under $\tilde{g}$ (if it is still present), but residing at the head of the queue under $g$, at time $S_{x+1}$.

For $L \leq x<B$,

$$
\begin{gathered}
F_{\beta}=\left\{S_{x-L+1}<A_{1}<S_{x-L+2} ; S_{x+1}<A_{2} ; U_{1}^{\phi}>p ; U_{1} \in(q, \hat{q}] ;\right. \\
U_{m}^{\prime} \leq \mu\left(X\left(S_{m}\right)\right) / \bar{\mu}, m=1, \ldots, x+1, m \neq x-L+2 ; \\
\left.U_{m}^{\prime} \in\left(\mu\left(X\left(S_{m}\right)\right) / \bar{\mu}, \mu\left(\tilde{X}\left(S_{m}\right)\right) / \bar{\mu}\right], m=x-L+2\right\},
\end{gathered}
$$

which, under coupling $\mathcal{C}$, results in the following occurrences, in the order presented.

1. The queue size under both $g$ and $\tilde{g}$ decreases from $x+1$ to $L$, and $C_{0}$ moves to position $L$ in both cases.

2. A controlled customer enters $Q_{\mathrm{S}}$ under $\tilde{g}$ but not under $g$; the resulting queue sizes are $L$ and $L+1$ under $g$ and $\tilde{g}$, respectively, and $C_{0}$ remains at position $L$ in both cases.

3. A departure occurs under $\tilde{g}$ but not under $g$. This results in the queue size being equal to $L$ in both cases, $C_{0}$ remaining at position $L$ under $g$, and $C_{0}$ moving to position $L-1$ (or even exiting the system if $L=1$ ) under $\tilde{g}$. 
4. A further $L-1$ departures occur under both $g$ and $\tilde{g}$ before the second arrival, resulting in $C_{0}$ leaving under $\tilde{g}$ (if it is still present), but residing at the head of the queue under $g$, at time $S_{x+1}$.

Define

$$
F_{\zeta}=\mathbf{1}_{\{x<L\}} F_{\alpha}+\mathbf{1}_{\{x \geq L\}} F_{\beta} .
$$

By conditioning on $F_{\zeta}$, it is easy to establish that $\mathrm{E}\left[v_{0}\right]>\mathrm{E}\left[\tilde{v}_{0}\right]$, and (ii) follows.

Note that, in the above proof, for clarity of exposition $\mu\left(x_{m}\right)$ and $\mu\left(\tilde{x}_{m}\right)$ have been written more explicitly as $\mu\left(X\left(S_{m}\right)\right)$ and $\mu\left(\tilde{X}\left(S_{m}\right)\right)$, in order to avoid confusion with the ' $x$ ' that appears in the indexing of the random variables.

We next show that, when other controlled customers adhere to the decision rule $[g]$, the expected sojourn time of a customer in $Q_{\mathrm{S}}$, for particular entry states $x$, is a continuous function of $g$. This result is established under the proviso that the following, not very restrictive, condition holds.

Condition 1. (Stability condition.) Under system $\mathrm{g}$, there exists a bound $D_{x}$ such that

$$
\mathrm{E}\left[\sum_{k=1}^{\infty} \mathbf{1}_{\left\{A_{k} \leq v_{0}\right\}}\right] \leq D_{x}
$$

uniformly in $g \in[0, B]$.

This condition says that the expected number of arrival instants that occur during the sojourn of $C_{0}$ in $Q_{\mathrm{S}}$, when controlled customers adopt the threshold decision rule [ $g$ ], is bounded above by $D_{x}$, over all $g \in[0, B]$. This condition will certainly be satisfied in the case in which the interarrival times, which are given by the $M_{i}$, are exponential. To see this, consider a system similar to $g$ except that its service rate is always $\mu(1)$. We flag quantities that are associated with this system with a superscript ' $*$ '. Under a coupling similar to $\mathcal{C}$ between this system and $g$, it can be shown that $v_{0} \leq v_{0}^{*}$ for all $g \in[0, B]$. However, it is also clear that $v_{0}^{*}$ only depends on the $N_{j}$ and the $U_{l}^{\prime}$ and, therefore, is independent of the arrival instants $A_{k}$.

Hence,

$$
\begin{aligned}
\mathrm{E}\left[\sum_{k=1}^{\infty} \mathbf{1}_{\left\{A_{k} \leq v_{0}\right\}}\right] & \leq \mathrm{E}\left[\sum_{k=1}^{\infty} \mathbf{1}_{\left\{A_{k} \leq v_{0}^{*}\right\}}\right] \\
& =\int_{0}^{\infty} \mathrm{E}\left[\sum_{k=1}^{\infty} \mathbf{1}_{\left\{A_{k} \leq t\right\}}\right] f_{v_{0}^{*}}(t) \mathrm{d} t \\
& =\lambda \int_{t=0}^{\infty} t f_{v_{0}^{*}}(t) \mathrm{d} t \\
& =\lambda \mathrm{E}\left[v_{0}^{*}\right]
\end{aligned}
$$

where $f_{v_{0}^{*}}(\cdot)$ is the probability density function of $v_{0}^{*}$. However, $\mathrm{E}\left[v_{0}^{*}\right]$ is just the expected value of the sum of $x+1$ i.i.d. exponential random variables, each with mean $\mu(1)^{-1}$. Thus,

$$
\mathrm{E}\left[\sum_{k=1}^{\infty} \mathbf{1}_{\left\{A_{k} \leq v_{0}\right\}}\right] \leq \lambda \frac{x+1}{\mu(1)} .
$$


Lemma 6. Suppose that the stability condition holds. Then, for every $k \in \mathbb{N}$ and $x \in$ $\{0,1, \ldots, B-1\}, V_{k}\left(x,[g]^{\infty}\right)$ is continuous in $g \in[0, B]$.

Proof. Without loss of generality, and to be specific, consider customer $C_{0}$ and the systems $g$ and $\tilde{g}$ under coupling $\mathcal{C}$, with the additional restrictions that $g=L+q$ and $\tilde{g}=L+\tilde{q}$, $0 \leq q<\tilde{q} \leq 1$. Define

$$
k_{0}=\inf \left\{k \in \mathbb{Z}^{+}: A_{k}<v_{0}, X\left(A_{k}\right)=L, U_{k} \in(q, \tilde{q}], U_{k}^{\phi}>p\right\},
$$

where inf $\varnothing:=\infty$. Indeed, if $k_{0}=\infty$ then $v_{0}=\tilde{v}_{0}$. For $k_{0}=k<\infty$, we have $\tilde{v}_{0}>A_{k}$ and

$$
\begin{aligned}
\mathrm{E}\left[\tilde{v}_{0}-v_{0} \mid k_{0}=k\right] & =\mathrm{E}\left[\tilde{v}_{0}-A_{k} \mid k_{0}=k\right]-\mathrm{E}\left[v_{0}-A_{k} \mid k_{0}=k\right] \\
& \leq(L+1) / \mu(1) .
\end{aligned}
$$

This follows from the fact that, on the first line, the first term on the right-hand side is bounded above by the expected time taken to serve $L+1$ customers at the slowest possible rate of $\mu(1)$, and from the fact that the second term on the right-hand side is bounded below by 0 .

It is easy to deduce that $\left\{A_{k}<v_{0}\right\}$ is independent of $\left\{U_{k} \in(q, \tilde{q}]\right\}$ (noting that the former is equivalent to $\left.\left\{Z\left(A_{k}\right)>0\right\}\right)$. Therefore,

$$
\begin{aligned}
\mathrm{P}\left(k_{0}=k\right) & \leq \mathrm{P}\left(A_{k}<v_{0}, U_{k} \in(q, \tilde{q}]\right) \\
& =\mathrm{P}\left(A_{k}<v_{0}\right) \mathrm{P}\left(U_{k} \in(q, \tilde{q}]\right) \\
& =(\tilde{q}-q) \mathrm{P}\left(A_{k}<v_{0}\right)
\end{aligned}
$$

and, hence,

$$
\begin{aligned}
\mathrm{E}\left[\tilde{v}_{0}-v_{0}\right] & \leq \frac{L+1}{\mu(1)} \sum_{k=1}^{\infty} \mathrm{P}\left(k_{0}=k\right) \\
& \leq(\tilde{q}-q) \frac{L+1}{\mu(1)} \sum_{k=1}^{\infty} \mathrm{P}\left(A_{k}<v_{0}\right) .
\end{aligned}
$$

By the monotone convergence theorem, $\sum_{k=1}^{\infty} \mathrm{P}\left(A_{k}<v_{0}\right)$ can be expressed as

$$
\mathrm{E}\left[\sum_{k=1}^{\infty} \mathbf{1}_{\left\{A_{k}<v_{0}\right\}}\right] \text {. }
$$

Furthermore, observe that $\tilde{q}-q=\tilde{g}-g$. Thus,

$$
\mathrm{E}\left[\tilde{v}_{0}-v_{0}\right] \leq(\tilde{g}-g) \frac{L+1}{\mu(1)} D_{x},
$$

as required.

\section{Structure and existence of the Nash equilibrium}

In this section, we first explore the required structure of any candidate symmetric Nash equilibrium policy (SNEP) within the $\mathbb{T}^{\infty}$ class. We then go on to prove that there exist a finite number of SNEPs within this class, and that at least one of these is characterized by a nonrandomized threshold. 
Lemma 7. Suppose that $\pi \in \mathbb{T}^{\infty}$. Then

(a) any decision rule of $C_{k}$ optimal against $\pi$ must be a threshold decision rule, and

(b) the set of decision rules of $C_{k}$ optimal against $\pi$, i.e. $\mathbb{U}_{k}(\pi)$, can be found in the following way, where $\hat{L}:=\min \left\{L \in \mathbb{Z}^{+}: V_{k}(L, \pi) \geq \theta\right\}$ :

(i) if $V_{k}(\hat{L}, \pi)=\theta$ then $\mathbb{U}_{k}(\pi)=\{[\hat{L}, q]: 0 \leq q \leq 1\}$;

(ii) otherwise $\mathbb{U}_{k}(\pi)=\{[\hat{L}, 0]\}$.

Proof. The proof follows from the definition of an optimal decision rule and the monotonicity result of Lemma 2. Since $V_{k}(L, \pi) \geq(L+1) / \bar{\mu} \rightarrow \infty$ as $L \rightarrow \infty, \hat{L}$ is well defined.

In the following discussion, the best response map of an arbitrary controlled customer $C^{(k)}$, say, is constructed against the background of other controlled customers adhering to the policy $[g]^{\infty}$. For simplicity, and without loss of generality, we construct this map for customer $C_{0}$ : this is as if to say that $C^{(k)}$ corresponds to $C_{0}$ in the overall arrival stream.

We define the mapping $l(\cdot)$ (as introduced in [2] but with a slight modification) as follows. For $g \in[0, B]$, let

$$
l(g)=\min \left\{n \in \mathbb{N}: n<B, V_{0}\left(n,[g]^{\infty}\right) \geq \theta\right\}
$$

with $\min \varnothing:=B$. Since $V_{0}(x, \cdot) \geq(x+1) / \bar{\mu} \rightarrow \infty$ as $x \rightarrow \infty, l(\cdot)$ is well defined. Furthermore, let $g_{1}, g_{2}, \ldots, g_{J}$ be the points of discontinuity of $l(g)$ for $g \in[0, B]$, where

$$
0=: g_{0} \leq g_{1}<g_{2}<\cdots<g_{J-1}<g_{J} \leq g_{J+1}:=B .
$$

Notice that $g_{1}=0$ if there is a point of discontinuity at the origin, and that $g_{J}=B$ if there is one at $B$ whenever $B$ is finite.

Following methodology similar to that in [2], we define the point-to-set mapping

$$
G^{*}(g):[0, B] \rightarrow 2^{[0, B]}
$$

as

$$
G^{*}(g)=\left\{g^{\prime} \in[0, B]:\left[g^{\prime}\right] \text { is optimal for } C_{0} \text { against }[g]^{\infty}\right\} .
$$

Since $[g]^{\infty}$ is a member of $\mathbb{T}^{\infty}$, we can invoke Lemma 7 to deduce that $G^{*}(\cdot)$ is given by

$$
G^{*}(g)= \begin{cases}\{l(g)+q, 0 \leq q \leq 1\} & \text { if } V_{0}\left(l(g),[g]^{\infty}\right)=\theta, l(g)<B, \\ l(g) & \text { if } V_{0}\left(l(g),[g]^{\infty}\right)>\theta, l(g)<B, \\ B & \text { if } l(g)=B .\end{cases}
$$

By the aforementioned properties of $V_{0}\left(\cdot,[g]^{\infty}\right)$, and provided that Condition 1 holds, it can easily be deduced that

$$
l(g)= \begin{cases}l(0), & 0 \leq g \leq 1, \\ l(0)+j, & g>1, g_{j}<g \leq g_{j+1} .\end{cases}
$$

Thus, $G^{*}(g)$ can be re-expressed as

$$
G^{*}(g)= \begin{cases}l(0) & \text { if } g<g_{1}, \\ {[l(0)+j-1, l(0)+j]} & \text { if } g=g_{j}, j=1, \ldots, J, \\ {[l(0), l(0)+1]} & \text { if } g_{0}=g_{1} \text { and } g \leq 1, \\ l(0)+j & \text { if } g_{j}<g<g_{j+1}, j=2, \ldots, J, \\ l(0)+1 & \text { if } g_{0}<g_{1}<g<g_{2}, \text { or } g_{0}=g_{1} \text { and } 1<g<g_{2} .\end{cases}
$$


Observe that the graph of $G^{*}(\cdot)$ is staircase arc-connected and nondecreasing whenever $g_{0}<g_{1}$; a similar structure will hold when $g_{0}=g_{1}$, except that we have a rectangular region with bottom-left and top-right coordinates given by $(0, l(0))$ and $(1, l(0)+1)$, respectively.

Define the map $H(g):=G^{*}(g)-g$, with the same domain as $G^{*}(\cdot)$. Here, $G^{*}(g)-g$ is taken to mean $\left[\min \left\{G^{*}(g)\right\}-g, \max \left\{G^{*}(g)\right\}-g\right]$. The graph of the map $H(\cdot)$ has a 'sawtooth-like' structure except in the case that $g_{0}=g_{1}$, when this is modified to include a rhombus on the interval $[0,1]$ with corners at $(0, l(0)),(0, l(0)+1),(1, l(0)-1)$, and $(1, l(0))$. We shall employ this construction in the proof of the following theorem.

Theorem 1. Suppose that Condition 1 holds. Then, in the class of policies $\mathbb{T}^{\infty}$,

(i) there exist a finite number of SNEPs, and

(ii) at least one of the SNEPs is nonrandomized.

Proof. The thresholds associated with the SNEPs correspond to the zeros of the map $H(\cdot)$. By Lemma 5(i), and using the fact that $l(0) \geq 1$, we have $\min \{H(g)\}>0$ for all $g \in[0,1)$. Thus, we restrict our discussion to $g \in[1, B]$.

Suppose that $B$ is finite. Then, since $\min \left\{G^{*}(1)\right\} \geq 1$ and $\max \left\{G^{*}(B)\right\} \leq B$, we have $\min \{H(1)\} \geq 0$ and $\max \{H(B)\} \leq 0$. Thus, by the structure of the graph of $H(\cdot)$, and the intermediate value theorem, there must exist a $g^{*} \in[1, B]$ for which $0 \in H\left(g^{*}\right)$.

Now suppose that $B$ is infinite. Notice that $V_{0}\left(x,[g]^{\infty}\right)$ is bounded below by $(x+1) / \bar{\mu}$, which is independent of $g$, and that $(x+1) / \bar{\mu} \rightarrow \infty$ as $x \rightarrow \infty$. Thus, there exists some $n \in \mathbb{Z}^{+}$for which $V_{0}\left(n,[g]^{\infty}\right)>\theta$ for all $g \geq 0$. This implies that $l(g) \leq n$ for all $g \geq 0$ and, so, the number of (vertical) jump points of $G^{*}(\cdot)$ (and $H(\cdot)$ ), which is given by $J$, must be finite. Therefore, $G^{*}(g)=l\left(g_{J}\right)+1<\infty$ for all $g>g_{J}$, which implies that $H(g)<0$ for all $g$ sufficiently large. From this, together with the fact that $\min \{H(1)\} \geq 0$, it follows that there exists a $g^{* *} \in[1, \infty)$ such that $0 \in H\left(g^{* *}\right)$.

As remarked earlier, since the number $J$ of vertical jumps is finite, the number of zeros of $H(\cdot)$ is bounded (for $B$ finite and infinite) and, so, finiteness of the number of SNEPs follows, establishing part (i).

Now suppose, in contradiction to part (ii), that there are no nonrandomized SNEPs. By part (i), at least one randomized SNEP exists. Such a point must correspond to a jump point of $H(\cdot)$. Indeed, an SNEP occurs at $g=g_{m}$ if and only if a zero that is strictly interior to the vertical part of the graph at $g_{m}$, i.e. $\min \left\{H\left(g_{m}\right)\right\}<0<\max \left\{H\left(g_{m}\right)\right\}$, also occurs. However, we know that $\min \{H(1)\} \geq 0$; thus, by the intermediate value theorem, there exists at least one point $g^{\prime} \in\left[1, g_{m}\right.$ ), on a diagonal section of the graph (which is taken to include corners), for which $0 \in H\left(g^{\prime}\right)$. Any such point must correspond to a nonrandomized threshold, providing the contradiction required for part (ii).

\section{Computation of the SNEPs}

The computation of the SNEPs is considered in this section. It will be assumed that the stability condition (Condition 1) holds throughout. As usual, attention will be restricted to customer $C_{0}$, although the argument extends easily to any $C_{k}$ with only minor changes in the subscript indexing. The procedures outlined require the calculation of $V_{0}\left(x,[g]^{\infty}\right)$ for various values of $x$ and $g$, the details of which have been deferred to Appendix A. 


\subsection{Evaluating the jump points of $G^{*}(\cdot)$}

The lowest point of the graph of $G^{*}(\cdot)$ at the origin is given by $l(0)$. The number of jump points is given by

$$
J=\max \left\{j \in \mathbb{J}: V_{0}\left(l(0)+j-1,[0]^{\infty}\right) \geq \theta, V_{0}\left(l(0)+j-1,[B]^{\infty}\right) \leq \theta\right\},
$$

with $\max \varnothing:=0$, where $\mathbb{J}:=\{1,2, \ldots, B-l(0)\}$. The jump points $g_{j}$ satisfy

$$
V_{0}\left(l(0)+j-1,\left[g_{j}\right]^{\infty}\right)=\theta
$$

for $j=1, \ldots, J$.

From the monotonicity and continuity of $V_{0}\left(\cdot,[g]^{\infty}\right)$, the $j$ th equation will have a solution if and only if

$$
V_{0}\left(l(0)+j-1,[0]^{\infty}\right) \geq \theta \quad \text { and } \quad V_{0}\left(l(0)+j-1,[B]^{\infty}\right) \leq \theta .
$$

In the case that $B=\infty$, we might more accurately replace this latter condition by

$$
\lim _{B \rightarrow \infty} V_{0}\left(l(0)+j-1,[B]^{\infty}\right) \leq \theta .
$$

These observations provide the basis for a systematic procedure for evaluating the $g_{j}$.

\subsection{Finding the SNEPs}

If an SNEP exists at $g^{*} \in\left(g_{j}, g_{j+1}\right)$ for $j=0,1, \ldots, J$, then the line of unit slope intersects the graph of $G^{*}(\cdot)$ on the horizontal section interior to $g_{j}$ and $g_{j+1}$. Indeed, the height of that section is $l(0)+j$ and, so, we deduce that $g^{*}$ is an SNEP in this location if and only if $g_{j}<l(0)+j<g_{j+1}$; in fact, $g^{*}=l(0)+j$.

On the other hand, $g^{*}=g_{j}$ for some $j=1,2, \ldots, J$ if and only if the line of unit slope intersects the vertical section of the graph of $G^{*}(\cdot)$ at $g_{j}$, i.e. $g_{j} \in[l(0)+j-1, l(0)+j]$. So, with knowledge of the $g_{j}$, these observations again provide the basis for a systematic procedure for finding the SNEPs within the $\mathbb{T}^{\infty}$ class.

\section{Behaviour of a dynamic learning scheme}

Consider a dynamic learning scheme in which each customer bases their joining decision on data collected by a central entity prior to its arrival at the system. Here it is assumed that the buffer size, $B$, is finite, and we consider a service rate function of the form

$$
\mu(x)=\bar{\mu}\left\{1-\frac{a}{(x+b)^{c}}\right\}, \quad x=1, \ldots, B,
$$

where $0<a \leq 1, b>0$, and $c>0$. We insist upon the presence of uncontrolled arrivals, in the sense that $p$ is strictly positive.

Under this scheme, each controlled customer follows the decision rule

$$
\text { join } Q_{\mathrm{S}} \text { at time } t \text { with probability } S_{\varepsilon}\left(\theta-\widehat{V}_{t}(X(t))\right),
$$

where $\varepsilon$ is a small positive parameter, $S_{\varepsilon}$ is an increasing function with $S_{\varepsilon}(x)=0$ for $x \leq-\varepsilon$ and $S_{\varepsilon}(x)=1$ for $x \geq \varepsilon$, and $\widehat{V}_{t}(x)$ is the empirical average (sample-mean) sojourn time of all customers who have exited $Q_{\mathrm{S}}$ by time $t$, but who entered it when the queue length was $x$. 

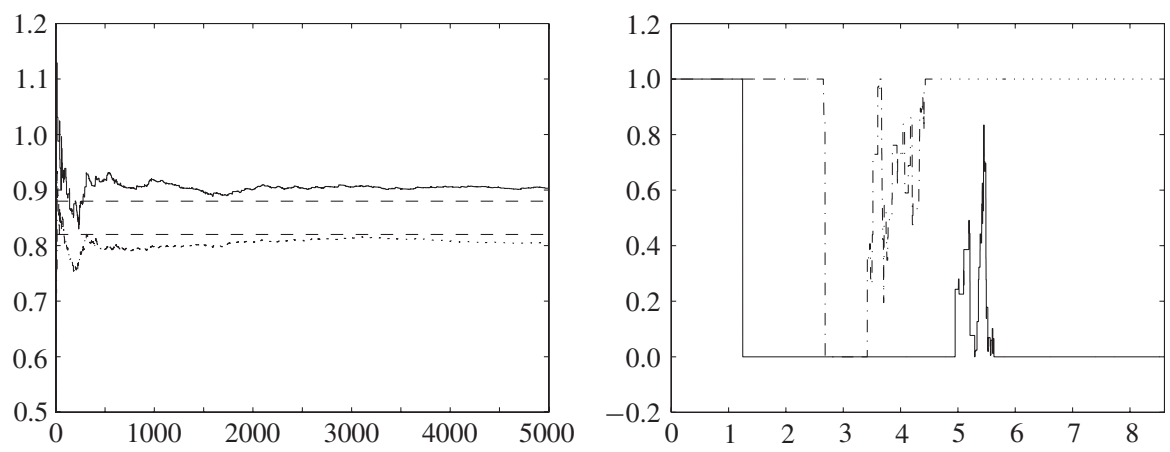

FIGURE 1: The left-hand diagram shows a plot of the empirical average $\widehat{V}_{t}(x)$, against time, for entry states $x=6$ (dash-dot line) and $x=7$ (solid line), also showing the bands at $\theta \pm \varepsilon$ (dashed lines). The right-hand diagram shows a plot of entrance probabilities for controlled customers, against the log of time, for entry states $x=6$ (dash-dot line) and $x=7$ (solid line). (See Simulation 1.)

The capacity for this scheme to 'learn' the Nash equilibria of the system under the stationary game is investigated using simulation. The examples are chosen to explore, in relative terms, three different regimes: (a) a small state space and a slowly varying service rate, (b) a large state space and a service rate quickly approaching the upper bound, and (c) a large state space and a slowly varying service rate.

Simulation 1. In this example, the arrivals are simulated to form a Poisson process with rate $\lambda=9$. The buffer size $B$ is set equal to 10 , with the service rate function $\mu(x)$, for $x=$ $1, \ldots, 10$, being specified by the parameter settings $\bar{\mu}=10, a=0.7, b=0.05$, and $c=1$. The parameter $p$, controlling the entrance of uncontrolled arrivals into the system, is set equal to 0.25 . Also, the quality-of-service parameter $\theta$ is set equal to 0.85 and $S_{\varepsilon}(\cdot)$ is chosen to correspond to the uniform cumulative distribution function on the interval $(\theta-\varepsilon, \theta+\varepsilon)$.

Under these parameter settings, it can be deduced that $l(0)=6$ and $g_{1}=1.8799$, with the number $J$ of jumps of the graph of $G^{*}(\cdot)$ being equal to 1 . There is a unique SNEP in the class $\mathbb{T}^{\infty}$, located at $g^{*}=7$. A simulation of this system is performed over a horizon length of 5000 time units, with $\varepsilon$ equal to 0.03 . The left-hand diagram of Figure 1 shows a plot of $\widehat{V}_{t}(x)$ against time for entry states 6 and 7, along with horizontal lines at $\theta-\varepsilon$ and $\theta+\varepsilon$. From a very early stage in the simulation, $\widehat{V}_{t}(x)$ stays well below $\theta-\varepsilon$ for entry states $x=0, \ldots, 5$, and stays well above $\theta+\varepsilon$ for states $x=8$ and $x=9$. For comparison, we remark that $V_{0}\left(6,[7]^{\infty}\right)$ and $V_{0}\left(7,[7]^{\infty}\right)$ are found to be 0.7959 and 0.9028 , respectively. A plot of the entrance probabilities against the natural logarithm of time, under the learning rule, for entry states 6 and 7 is depicted in the right-hand diagram of Figure 1. The results of this experiment appear to support the hypothesis that the system-wide statistics converge to those corresponding to the stationary threshold at $g^{*}=7$.

Simulation 2. For this example, the parameter settings are exactly the same as those in Simulation 1, except that the service rate function is specified by the parameters $a=0.9, b=0.05$, and $c=3$ and the size of the state space is somewhat larger, with $B=25$. The graph of $G^{*}(\cdot)$ again has one jump, with $l(0)=7$ and $g_{1}=1.0666$, and, so, we deduce that a single SNEP resides within the class $\mathbb{T}^{\infty}$, at $g^{*}=8$.

Again, the simulation is performed over 5000 time units. Empirical averages for $x=$ $0, \ldots, 6$ stay well below $\theta-\varepsilon$ and, for $x=9,10, \ldots, 24$, well above $\theta+\varepsilon$. Figure 2 depicts 

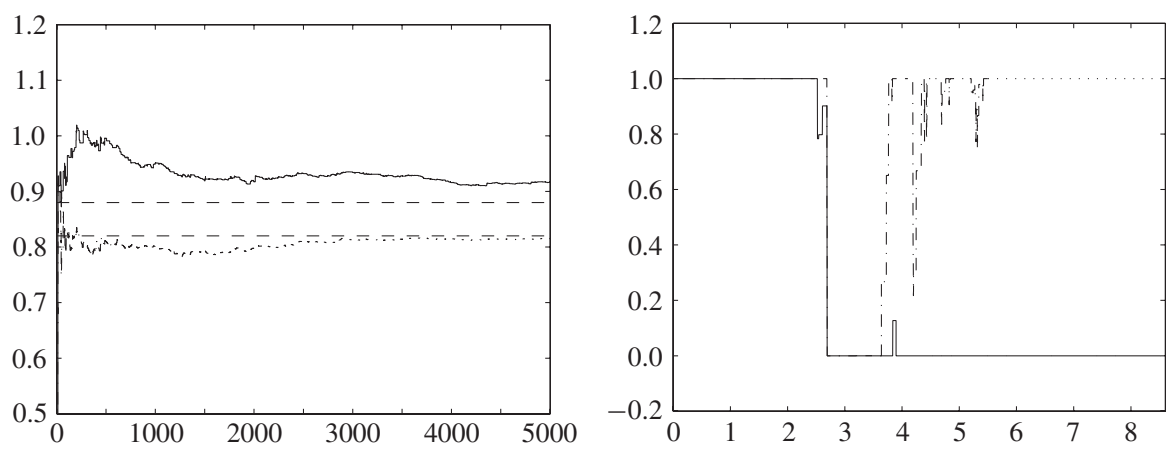

FIGURE 2: The left-hand diagram shows a plot of the empirical average $\widehat{V}_{t}(x)$, against time, for entry states $x=7$ (dash-dot line) and $x=8$ (solid line), also showing the bands at $\theta \pm \varepsilon$ (dashed lines). The right-hand diagram shows a plot of entrance probabilities for controlled customers, against the log of time, for entry states $x=7$ (dash-dot line) and $x=8$ (solid line). (See Simulation 2.)
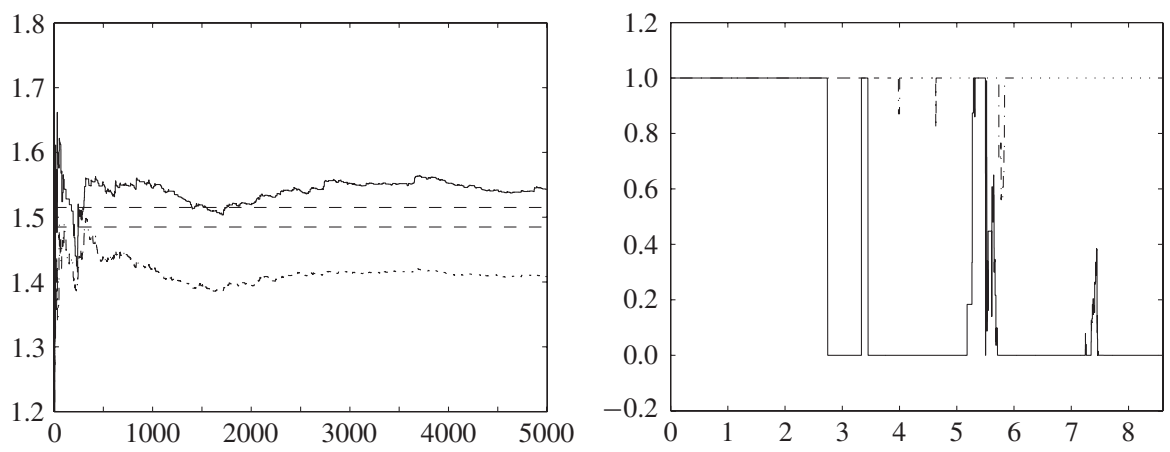

FIGURE 3: The left-hand diagram shows a plot of the empirical average $\widehat{V}_{t}(x)$, against time, for entry states $x=11$ (dash-dot line) and $x=12$ (solid line), also showing the bands at $\theta \pm \varepsilon$ (dashed lines). The right-hand diagram shows a plot of entrance probabilities for controlled customers, against the log of time, for entry states $x=11$ (dash-dot line) and $x=12$ (solid line). (See Simulation 3.)

empirical averages for the states $x=7$ and $x=8$ in the left-hand diagram, and entrance probabilities in the right-hand diagram. The values of $V_{0}\left(7,[8]^{\infty}\right)$ and $\left.V_{0}\left(8,{ }^{[8}\right]^{\infty}\right)$ are 0.8049 and 0.9046 , respectively.

Simulation 3. In this example, $\lambda=9$ and the service rate is specified by the parameters $\bar{\mu}=10, a=1, b=1$, and $c=0.8$, with $B=25$. The parameters $\theta$ and $\varepsilon$ are equal to 1.5 and 0.015 , respectively. The graph of $G^{*}(\cdot)$ displays one jump, with $l(0)=12$ and $g_{1}=13.1683$, and, so, we deduce that a single SNEP resides within the class $\mathbb{T}^{\infty}$, at $g^{*}=12$.

Again, the simulation is performed over 5000 time units. We observe that the empirical averages for $x=0, \ldots, 10$ stay well below $\theta-\varepsilon$ and, for $x=13, \ldots, 24$, well above $\theta+\varepsilon$. Figure 3 depicts empirical averages for the states $x=11$ and $x=12$ in the left-hand diagram, and entrance probabilities in the right-hand diagram. The values of $V_{0}\left(11,[12]^{\infty}\right)$ and $V_{0}\left(12,[12]^{\infty}\right)$ are 1.3998 and 1.5116 , respectively. 


\section{Conclusions}

In this paper, we have established the existence of a certain symmetric Nash equilibrium policy for customers joining a queue with load-increasing service rate, based on the observed queue size on arrival at the system. We proved that at least one of the Nash equilibria is nonrandomized; this is a somewhat different phenomenon to that exhibited in [2], [4], and [5]. Constancy in $g$ of the expected sojourn time in the region [0,1], and strict monotonicity in $[1, B]$, was established as an essential stepping stone for the game-theoretic results. (The constancy in $[0,1]$ appears to have been overlooked in the author's related previous papers. However, conditions analogous to $\mu(1)^{-1}<\theta$, which imply that it is worth entering the system when it is completely empty, suggest that the consequences of any such oversight are only very slight.)

Simulation experiments suggest that, when a unique (nonrandomized) SNEP of the stationary game exists in the class $\mathbb{T}^{\infty}$, quantities such as the empirical averages and simulated entrance probabilities, under the learning rule, show a close correspondence to expected sojourn times and entrance probabilities under the SNEP in the associated stationary game. Convergence and stability properties in the case of multiple Nash equilibria are not well understood at this stage; however, simulation experiments appear to suggest that the SNEPs are viable poles of attraction and provide a rough guide to the operating points of the system.

\section{Acknowledgements}

This work was carried out, in part, while the author was visiting the School of Mathematical Sciences at Queen Mary, University of London. The author acknowledges the financial support of the Nuffield Foundation, under grant number NAL/00721/G, and is grateful to an anonymous referee for comments on improving the simulation examples.

\section{Appendix A. Evaluating $V_{0}\left(\cdot,[g]^{\infty}\right)$}

Via a set of linear equations, we here present a procedure for calculating the expected sojourn time of a customer entering $Q_{\mathrm{S}}$ under the symmetric equilibrium policy $[g]^{\infty}$, with $g=L+q$, and with buffer size $B$. We assume that the arrival process consists of the superposition of two independent Poisson processes: (a) the controlled arrival process, with rate $\lambda_{\mathrm{c}}$, in which customers are governed by the decision rule $[g]$, and (b) the uncontrolled arrival process, with rate $\lambda_{\mathrm{u}}$, in which customers always join the system, provided that the buffer size is not exceeded.

Let $R(x, y)$, with $R(x, 0):=0$, be the expected remaining sojourn time of a customer who has precisely $y-1$ customers ahead of it in the queue, when the queue length is $x$ (and $0<y \leq x) . R(x, y)$ satisfies the following set of linear equations, where $\alpha:=\lambda+\bar{\mu}$ :

$$
\begin{array}{lc}
R(x, y)=\frac{1}{\alpha}[1+\mu(x) R(x-1, y-1)+(\bar{\mu}-\mu(x)) R(x, y)+\lambda R(x+1, y)], & 0<y \leq x<L \leq B ; \\
R(L, y)=\frac{1}{\alpha}\left[1+\mu(L) R(L-1, y-1)+\left\{(\bar{\mu}-\mu(L))+\lambda_{\mathrm{c}}(1-q)\right\} R(L, y)\right. \\
\left.+\left(\lambda_{\mathrm{c}} q+\lambda_{\mathrm{u}}\right) R(L+1, y)\right], & 0<y \leq L<B ; \\
R(x, y)=\frac{1}{\alpha}\left[1+\mu(x) R(x-1, y-1)+\left\{\bar{\mu}-\mu(x)+\lambda_{\mathrm{c}}\right\} R(x, y)+\lambda_{\mathrm{u}} R(x+1, y)\right], & 0<y \leq x, L+1 \leq x<B ;
\end{array}
$$


and

$R(B, y)=\frac{1}{\alpha}[1+\mu(B) R(B-1, y-1)+\lambda R(B, y)], \quad 0<y \leq B$.

It is easily seen that $V_{0}\left(x,[g]^{\infty}\right)=R(x+1, x+1)$.

\section{References}

[1] Altman, E. And Shimkin, N. (1996). Learning and equilibrium in processor sharing systems. Res. Rep. EE1113, Department of Electrical Engineering, Technion.

[2] Altman, E. And Shimkin, N. (1998). Individual equilibrium and learning in processor sharing systems. Operat. Res. 46, 776-784.

[3] Ben-Shahar, I., Orda, A. And Shimkin, N. (2000). Dynamic service sharing with heterogeneous preferences. Queueing Systems 35, 83-103.

[4] Brooms, A. C. (2000). Individual equilibrium dynamic routing in a multiple server retrial queue. Prob. Eng. Inf. Sci. 14, 9-26.

[5] Brooms, A. C. (2003). Assessing the performance of a shared resource: simulation vs. the Nash equilibrium. In Keynote Papers, YOR13 (Bath, UK, 2003), ed. J. Taylor, The Operational Research Society, Birmingham, pp. 3-19.

[6] Buche, R. AND Kushner, H. J. (2000). Stochastic approximation and user adaptation in a competitive resource sharing system. IEEE Trans. Automatic Control 45, 844-853.

[7] Lippman, S. (1975). Applying a new device in the optimization of exponential queuing systems. Operat. Res. 23, 687-709.

[8] Lippman, S. A. and STIDHam, S. (1977). Individual versus social optimisation in exponential congestion systems. Operat. Res. 25, 233-247.

[9] NAOR, P. (1969). The regulation of queue size by levying tolls. Econometrica 37, 15-24.

[10] Yechiali, U. (1971). On optimal balking rules for the GI/M/1 queueing process. Operat. Res. 19, 349-370. 